\title{
STATIONARY SOLUTIONS OF THE GENERALIZED SMOLUCHOWSKI-POISSON EQUATION
}

\author{
ROBERT STAŃCZY \\ Instytut Matematyczny, Uniwersytet Wroctawski \\ pl. Grunwaldzki 2/4, 50-384 Wroctaw, Poland \\ E-mail: stanczr@math.uni.wroc.pl
}

\begin{abstract}
The existence of steady states in the microcanonical case for a system describing the interaction of gravitationally attracting particles with a self-similar pressure term is proved. The system generalizes the Smoluchowski-Poisson equation. The presented theory covers the case of the model with diffusion that obeys the Fermi-Dirac statistic.
\end{abstract}

1. Introduction. We consider the following nonlocal elliptic boundary value problem with given mass $M_{0}>0$ and the energy $E_{0} \in \mathbb{R}$ :

$$
\begin{aligned}
\Delta \varphi & =\theta^{d / 2} H^{-1}\left(\frac{c-\varphi}{\theta}\right) \text { in } \Omega, \\
\varphi & =0 \text { on } \partial \Omega, \\
M_{0} & =\int_{\Omega} \Delta \varphi d x \\
E_{0} & =\frac{d}{2} \int_{\Omega} \theta^{d / 2+1} P\left(\theta^{-d / 2} \Delta \varphi\right) d x-\frac{1}{2} \int_{\Omega}|\nabla \varphi|^{2} d x,
\end{aligned}
$$

where $P(z)$ is a given function, $H(z)$ is a primitive of $P^{\prime}(z) / z, \Omega \subset \mathbb{R}^{d}$ is a bounded domain with smooth boundary $\partial \Omega$, and finally the unknowns: $\theta$ is a positive constant temperature, $\varphi=\varphi(x)$ the gravitational potential and $c$ is some constant to be determined.

The solutions of the above problem can be regarded as the stationary solutions of the following parabolic-elliptic system, motivated by some astrophysical models of selfgravitating particles and derived by statistical mechanics approach:

$$
n_{t}=\nabla \cdot\left(\theta P^{\prime 2} \nabla n+n P^{\prime} \nabla \varphi\right) \quad \text { in } \Omega \times(0, \infty)
$$

2000 Mathematics Subject Classification: 35Q, 35J20, 35J65, 47J30, 82C21.

Key words and phrases: Chavanis-Sommeria-Robert model, Smoluchowski-Poisson equation, Fermi-Dirac statistics, variational methods.

The paper is in final form and no version of it will be published elsewhere. 


$$
\begin{aligned}
\Delta \varphi=n & & \text { in } \Omega \times(0, \infty), \\
\left(\theta P^{\prime 2} \nabla n+n P^{\prime} \nabla \varphi\right) \cdot \bar{\nu}=\varphi=0 & & \text { on } \partial \Omega \times(0, \infty), \\
n(0)=n_{0} \geq 0 & & \text { in } \Omega,
\end{aligned}
$$

where $n=n(x, t)$ is a nonnegative density of the particles inducing the gravitational potential $\varphi=\varphi(x, t)$. The equation (5) for $P^{\prime}=$ const and given exterior force $\nabla \varphi$ goes back to the paper [13] by the Polish physicist Marian Smoluchowski. Here the potential $\varphi$ is coupled with the density $n$ via the Poisson equation (6). This specific model was considered by Chavanis et al. in [8] and treated rigorously in a different framework in [2]. For the related papers see also the references in [14]-[17].

The proof of the existence of at least one solution for the problem (1)-(4) under a suitable assumptions on the data and the form of $P$ including the one obeying FermiDirac statistics is the main result of this paper. This will be obtained by the minimization of the neg-entropy functional $\mathcal{W}$ defined by

$$
\mathcal{W}(n, \theta)=\int_{\Omega}\left(n H-\left(\frac{d}{2}+1\right) P \theta^{d / 2}\right) d x,
$$

for the functions $n=n(x)=\Delta \varphi(x)$ from $L^{1+2 / d}(\Omega)$ satisfying constraints (3)-(4), where the functions $P(z), H^{\prime}(z)=P^{\prime}(z) / z$ depend on $z=n \theta^{-d / 2}$. The neg-entropy $\mathcal{W}$ plays here the role of a Lyapunov functional for the problem (5)-(8), (10), (11) (cf. [4]) from which we shall derive its stationary counterpart (1)-(4).

2. Derivation of the steady state problem. Note that due to the boundary condition (7) mass conservation

$$
M(n) \stackrel{\text { df }}{=} \int_{\Omega} n d x=M_{0}
$$

readily follows from the integration of (5) and together with (6) justifies (3).

The problem (5)-(8) can be considered in the canonical (isothermal) setting with the constant temperature $\theta$ or, as in our case, in the microcanonical (nonisothermal) setting, i.e. with the given energy and the temperature to be determined so that the energy relation

$$
E(n, \theta) \stackrel{\mathrm{df}}{=} \frac{d}{2} \int_{\Omega} \theta^{d / 2+1} P\left(n \theta^{-d / 2}\right) d x+\frac{1}{2} \int_{\Omega} n \Delta^{-1} n d x=E_{0}
$$

is satisfied where $\varphi=\Delta^{-1} n$ solves the Poisson equation $\Delta \varphi=n$ with the homogeneous Dirichlet boundary consition. This can be rephrased for the stationary problem as (4).

Multiplying (5) by $\theta H+\varphi$ and integrating by parts one obtains, for some constant $c$, the equation for the steady states

$$
\theta H+\varphi=c
$$

or, if the function $H$ is increasing, equivalently (1).

Note, that applying the Lagrange Multiplier Rule for $\mathcal{W}$ defined by (9) (or to be exact for $\mathcal{W}-\alpha E-\beta M$ ) we get the Euler-Lagrange equation (with the Gateaux derivative denoted as ')

$$
\mathcal{W}^{\prime}-\alpha E^{\prime}-\beta M^{\prime}=0
$$


with constraints (10) and (11), or equivalently computing the partial derivatives with respect to $n$ and $\theta$ accordingly,

$$
\begin{aligned}
H(z)-\alpha \Delta^{-1} n-\beta-\frac{d}{2} P^{\prime}(z)(1+\alpha \theta) & =0, \\
\left(\left(1+\frac{2}{d}\right) P(z)-P^{\prime}(z) z\right)(1+\alpha \theta) & =0,
\end{aligned}
$$

where $z=n \theta^{-d / 2}$ and (10)-(11) hold. Therefore, if $P$ is not a polytropic pressure density of order $1+2 / d$, i.e. $C z^{1+2 / d}$, then $\alpha=-1 / \theta$ and from the first equation we get the equation for the steady states, namely (12) with $c=\beta \theta$.

3. Overview of the known results for various pressure forms. Numerous pressure formulas coming from statistical mechanics of self-interacting particles have been collected in [4] including Maxwell-Boltzmann, Bose-Einstein, Fermi-Dirac and polytropic distributions as in the examples below. General approach to the entropy methods can be found in $[1]$.

ExAmple 1. In the Maxwell-Boltzmann case $P_{M B}=I$. This classical Boltzmann relation leads to the linear Brownian diffusion term $\Delta n$ in (5). Note that the steady states were fully analyzed in [6] and [7] as the critical points for the corresponding neg-entropy functional $\mathcal{W}$ on the space of functions with $\int_{\Omega} n \log n d x<\infty$ as $H(z)=\log z$. Therefore the functional setting is totally different from the examples requiring the power-like growth of $H(z)$ as presented below.

ExAmple 2. For the Fermi-Dirac distributions one has

$$
P_{F D}(z)=\frac{\mu}{d}\left(f_{d / 2} \circ f_{d / 2-1}^{-1}\right)\left(\frac{2}{\mu} z\right) .
$$

The function $f_{\alpha}$ denotes the Fermi integral of order $\alpha>-1$ defined by

$$
f_{\alpha}(z)=\int_{0}^{\infty} \frac{y^{\alpha} \mathrm{d} y}{\mathrm{e}^{y-z}+1} .
$$

This leads to a nonlinear diffusion in (5). Properties of Fermi integrals (17) (convexity, asymptotics, etc.) relevant to study the system (1)-(4) and (5)-(8) are collected in [2, Sec. 2], [3, Sec. 5] or [17, Appendix]. In this case the leading term coefficient, being the limit of $P_{F D}(z) z^{-1-2 / d}$ as $z \rightarrow \infty$, equals $p_{1}=\frac{2}{d+2}\left(\frac{d}{\mu}\right)^{2 / d}$, where $\mu=\eta_{0} 2^{d / 2} G \sigma_{d}^{2}$ and $G$ is the gravitational constant, $\sigma_{d}$ the measure of the unit sphere in $\mathbb{R}^{d}$ and $\eta_{0}$ a bound on the density in the $(x, v)$ phase space. The problem of the existence of steady states has been considered e.g. in [15] and [16].

EXAmPLE 3. An analogous construction is used to define the Bose-Einstein distributions which properties, however, differ from those of Fermi-Dirac ones. The pressure assumes the form

$$
P_{B E}(z)=\frac{\mu}{d}\left(g_{d / 2} \circ g_{d / 2-1}^{-1}\right)\left(\frac{2}{\mu} z\right),
$$

which again leads to a nonlinear diffusion in (5). Here $g_{\alpha}$ denotes the Bose-Einstein 
integral of order $\alpha>-1$ (cf. [4, Sec. 2]) defined, for $z<0$, by

$$
g_{\alpha}(z)=\int_{0}^{\infty} \frac{y^{\alpha} \mathrm{d} y}{\mathrm{e}^{y-z}-1} .
$$

In this case, however, due to the fact that, for $\alpha>0, G=\sup _{z<0} g_{\alpha}(z)$ is finite, $P_{\mathrm{BE}}$ could be defined only for $\frac{2}{\mu} \frac{n}{\theta^{d / 2}} \leq G$, and a totally different approach should be applied. EXAmple 4. Polytropes are classical equations of state of a gas

$$
P_{1+\gamma}(z)=\kappa_{\gamma} z^{1+\gamma}
$$

with a polytropic constant $\kappa_{\gamma}>0$ and $0<\gamma<2 / d$ so that $\frac{\partial p}{\partial \theta}>0$, which is physically a natural condition. The limit value $\gamma=2 / d$ leads to the pressure

$$
P_{1+2 / d}(z)=\kappa_{2 / d} z^{1+2 / d},
$$

independent of $\theta$. The limit case $\gamma \searrow 0$ corresponds to the Boltzmann density-pressure relation. The polytropic relations define evolution equations with nonlinear diffusions as, e.g., in the porous media equation (cf. [10]). Note that the steady states in this case are described by the same equation but with different boundary condition (the Neumann one) as for the model of chemotaxis (see [11] and [12] for the steady states and [9], [18] and [19] for the evolution problem).

A common feature for all the aforementioned examples is the dependence of the pressure $P$ on the ratio $n / \theta^{d / 2}$. Due to this property of the problem (5)-(8), (10) and (11), the neg-entropy functional exists (for the proof see [4]).

4. Main results. We shall use the following energy a priori estimates for the solutions of (1)-(4) (cf. [5]).

Lemma 4.1. For any $d \in\{2,3,4\}$ and $\int_{\Omega} n d x=M_{0}$ we have the estimate

$$
\left|\int_{\Omega} n \Delta^{-1} n d x\right| \leq C M_{0}^{1-2 / d} \int_{\Omega} n^{1+2 / d} d x .
$$

Proof. The proof follows directly from the Sobolev-Gagliardo-Nirenberg inequality.

Lemma 4.2. Let $\nu=4 /(d(4-d))$ for any $2 \leq d<4$. Assume that, for all $z \geq 0$,

$$
P(z) \geq p_{1} z^{1+2 / d} \text {. }
$$

Then, for any $\varepsilon$ with $\frac{d}{2} p_{1}>\varepsilon>0$ and any $n$ satisfying (11) with $\int_{\Omega} n d x=M_{0}$, the following estimate holds:

$$
E_{0}+C M_{0}^{1+\nu} \geq \max \left\{\varepsilon \int_{\Omega} n^{1+2 / d} d x,\left|\int_{\Omega} n \Delta^{-1} n d x\right|\right\} .
$$

Proof. The proof, analogous to the one of the previous lemma, makes use of assumption (22) (cf. [3] and [4]).

Now we are ready to formulate the main existence theorem.

TheOREM 4.3. Assume that $P$ is a $C^{1}$, nonnegative, increasing function satisfying (22) and, for some positive constants $p_{1}, C, M_{0}$,

$$
P^{\prime}(z) \geq p_{1},
$$




$$
\begin{aligned}
& \max \left\{P(z), z P^{\prime}(z)\right\} \leq C\left(1+z^{1+2 / d}\right), \\
& \left(P^{\prime}(z) z^{-2 / d}\right)^{\prime} \leq 0, \\
& \liminf _{z \rightarrow \infty}(H(z)-(d / 2+1) P(z) / z)>\mathcal{W}_{\min } / M_{0},
\end{aligned}
$$

where $\mathcal{W}_{\text {min }}=\inf _{M_{0}, E_{0}} \mathcal{W}$ is negative. Then the neg-entropy functional $\mathcal{W}$ attains its negative minimum $\mathcal{W}_{\text {min }}$ and therefore problem (1)-(4) possesses a solution, provided the data satisfies $d p_{1}>C M_{0}^{1-2 / d}$ for $d=4$ (for $d<4$ no smallness condition appears).

Proof. Using the Direct Method in the Calculus of Variations we shall show that the minimizing sequence $\left(n_{k}, \theta_{k}\right)$ converges to the minimizer of the functional $\mathcal{W}$ satisfying (3) and (4). Unfortunately due to assumptions (22), (25) the leading terms in (9) being of order $1+2 / d$ may cancel out and lower order terms might not provide any a priori estimates for the densities $n_{k}$. However, due to the energy constraint (4) and the estimate (22) we obtain, for $n_{k}=\Delta \varphi_{k}$, that

$$
\frac{d p_{1}}{2} \int_{\Omega} n_{k}^{1+2 / d} d x \leq E_{0}-\frac{1}{2} \int_{\Omega} n_{k} \Delta^{-1} n_{k} d x,
$$

which together with Lemma 4.1 gives, for sufficiently small data $\left(d p_{1}>C M_{0}^{1-2 / d}\right.$ for $2 \leq d \leq 2(1+\sqrt{2}))$, the required estimate for the $L^{1+2 / d}(\Omega)$ norm $\left|n_{k}\right|_{1+2 / d}$, i.e.

$$
\left(d p_{1}-C M_{0}^{1-2 / d}\right) \int_{\Omega} n_{k}^{1+2 / d} d x \leq 2 E_{0} .
$$

If we want to obtain the result for any mass (possibly large one) but only for $2 \leq d<4$, then we may apply Lemma 4.1 to the inequality (28) and get

$$
\frac{d p_{1}}{2} \int_{\Omega} n_{k}^{1+2 / d} d x \leq \frac{3}{2} E_{0}+C M_{0}^{1+\nu},
$$

or directly use the estimate $(23)$.

Thus, in any case, due to the boundedness of the $L^{1+2 d}$ norm of $n_{k}$ we can extract a weakly convergent subsequence still denoted $n_{k}$.

Then using another lower bound for the pressure $P(z) \geq p_{1} z$, which follows from (24), we get

$$
\frac{d p_{1}}{2} \theta_{k} M_{0} \leq E_{0}-\frac{1}{2} \int_{\Omega} n_{k} \Delta^{-1} n_{k} d x
$$

and using once again Lemma 4.1 and the bound for $\left|n_{k}\right|_{1+2 / d}$ we get the upper bound for $\theta_{k}$.

Next, we focus on showing that the sequence $\theta_{k}$ is bounded from below. We use a similar approach as in [5] and [14] where a priori bounds on the temperature for (5)-(8), (10), (11) were proved for negative initial values of the entropy $\mathcal{W}(0)<0$.

It follows from the assumption (27) that for $\mathcal{W}_{\min }<0$ and $\varepsilon>0$ such that $\varepsilon+$ $\mathcal{W}_{\text {min }} / M_{0}<0$, we can find $\delta>0$ such that for any $z \geq \delta$ we have

$$
R(z) \stackrel{\text { df }}{=} H(z) z-(d / 2+1) P(z) \geq z\left(\varepsilon+\mathcal{W}_{\min } / M_{0}\right) .
$$

Next, due to the assumption (24), fix some negative number $\mathcal{W}_{0} \leq \inf _{z \leq \delta} R(z)$. Then we 
obtain

$$
\begin{aligned}
\mathcal{W}\left(n_{k}, \theta_{k}\right) & =\left(\int_{\left\{n_{k} / \theta_{k}^{d / 2} \geq \delta\right\}}+\int_{\left\{n_{k} / \theta_{k}^{d / 2}<\delta\right\}}\right) \theta_{k}^{d / 2} R\left(\frac{n_{k}}{\theta_{k}^{d / 2}}\right) \mathrm{d} x \\
& \geq M_{0}\left(\varepsilon+\mathcal{W}_{\min } / M_{0}\right)+\mathcal{W}_{0}|\Omega| \theta_{k}^{d / 2} .
\end{aligned}
$$

This leads to the required bound $\theta_{k}^{d / 2} \geq \frac{\varepsilon M_{0}}{2|\Omega|\left(-\mathcal{W}_{0}\right)}$, since for any $k$ large enough we have $\mathcal{W}\left(n_{k}, \theta_{k}\right)-\mathcal{W}_{\min } \leq \varepsilon M_{0} / 2$.

Thus we can consider the subsequence $\left(n_{k}, \theta_{k}\right)$ weakly covergent to some element $\left(n_{0}, \theta_{0}\right)$. Therefore, by $(26)$ the convexity of $\mathcal{W}\left(\cdot, \theta_{0}\right)$ and continuity implies its weak lower semi-continuity, hence

$$
\liminf _{k \rightarrow \infty} \mathcal{W}\left(n_{k}, \theta_{0}\right) \geq \mathcal{W}\left(n_{0}, \theta_{0}\right) .
$$

Finally, from the estimates on $\left|n_{k}\right|_{1+2 / d}$ and, by the use of (25), on the derivative

$$
\frac{\partial \mathcal{W}}{\partial \theta}=\theta^{d / 2}\left(\frac{d}{2}\right)^{2} \int_{\Omega} P^{\prime}(z) z-(1+2 / d) P(z) d x,
$$

we obtain the equality

$$
\liminf _{k \rightarrow \infty} \mathcal{W}\left(n_{k}, \theta_{k}\right)=\liminf _{k \rightarrow \infty} \mathcal{W}\left(n_{k}, \theta_{0}\right)
$$

which guarantees that the minimum is attained at $\left(n_{0}, \theta_{0}\right)$.

Corollary 4.4. Assume that we have

$$
P(z)=p_{1} z^{1+2 / d}+o\left(z^{1+2 / d}\right) .
$$

with $o(z) / z \rightarrow 0$ as $z \rightarrow \infty$. Moreover, let the assumptions of Theorem 4.3 be satisfied except (27) to be replaced by

$$
\lim _{z \rightarrow \infty}\left(G(z)-(d / 2) z G^{\prime}(z)\right) \stackrel{\text { df }}{=} G_{0} \geq 0,
$$

where

$$
H^{\prime}(z)=h_{1}(2 / d) z^{2 / d-1}+G^{\prime}(z),
$$

with $G^{\prime}(z)=o\left(z^{2 / d-1}\right)$. Then the neg-entropy functional $\mathcal{W}$ attains its negative minimum and the problem (1)-(4) has at least one solution.

Proof. If (33) holds, then the highest order terms appearing in (27) cancel. Then

$$
\begin{aligned}
\lim _{z \rightarrow \infty} \frac{H(z) z-(d / 2+1) P(z)}{z} & =\lim _{z \rightarrow \infty}\left(H(z)-\frac{d}{2} z H^{\prime}(z)\right) \\
& =\lim _{z \rightarrow \infty}\left(G(z)-(d / 2) z G^{\prime}(z)\right) \stackrel{\mathrm{df}}{=} G_{0} \geq 0
\end{aligned}
$$

which guarantees (27). In particular, it should be noted that for the specific pressure forms we have to deal with the lower order term, e.g. $G^{\prime}(z)=g_{1} z^{\beta}+o\left(z^{\beta}\right)$ with some $\beta<2 / d-1$.

Corollary 4.5. The same statement as in Corollary 4.4 holds for the Fermi-Dirac pressure $P=P_{F D}$ defined in Example 2 in Section 3.

Proof. As was noted before we are left with the analysis of the lower order term $G^{\prime}(z)=$ $g_{1} z^{\beta}+o\left(z^{\beta}\right)$ with some $\beta<2 / d-1$. Namely, if $\beta<-1$ then $G_{0}=0$, e.g. for the 
Fermi-Dirac model. The other required properties of the Fermi-Dirac pressure follow from the papers that have been already cited in Example 2.

Final comments and remarks. It should be noted however, that the Euler-Lagrange equation was derived formally and in fact, holds only when the density of the minimizer $n_{0}$ is positive. To prove this one can argue by contradiction either as in [15] where fixed mass and the temperature were considered for Fermi-Dirac model or in [7] where mass and the energy were fixed for the case corresponding to Maxwell-Boltzmann distribution. If the density $n_{0}$ was positive on some set we could shift part of mass to zero level set decreasing thus the value of the entropy density (note that the absolute value of the entropy grows fastest in the neighbourhood of zero). The question arises: how to obtain this, keeping the energy value constant? Some arguments were provided for the linear case in [7] or for the Fermi-Dirac case with fixed mass in [15]. The full exposition of this argument in the microcanonical case for the Fermi-Dirac model lies beyond the scope of this paper.

Acknowledgements. The preparation of this paper was partially supported by the European Commission Marie Curie Host Fellowship for the Transfer of Knowledge "Harmonic Analysis, Nonlinear Analysis and Probability" MTKD-CT-2004-013389 and by the Polish Ministry of Science project N201 022 32/0902.

\section{References}

[1] A. Arnold, J. A. Carrillo, L. Desvillettes, J. Dolbeault, A. Jüngel, C. Lederman, P. A. Markowich, G. Toscani and C. Villani, Entropies and equilibria of many-particle systems: an essay on recent research, Monatshefte für Mathematik 142 (2004), 35-43.

[2] P. Biler, Ph. Laurençot and T. Nadzieja, On an evolution system describing self-gravitating Fermi-Dirac particles, Adv. Differential Equations 9 (2004), 563-586.

[3] P. Biler, T. Nadzieja and R. Stańczy, Nonisothermal systems of self-interacting FermiDirac particles, in: Nonlocal Elliptic and Parabolic Problems, P. Biler, G. Karch and T. Nadzieja (eds.), Banach Center Publications 66, Inst. Math., Polish Acad. Sci., Warszawa, 2004, 61-78.

[4] P. Biler and R. Stańczy, Parabolic-elliptic systems with general density-pressure relations, in: Variational Problems and Related Topics, M. Misawa and T. Suzuki (eds.), Sūrikaisekikenkyūsho Kōkyūroku 1405, Kyoto, 2004, 31-53.

[5] P. Biler and R. Stańczy, Nonlinear diffusion models for self-gravitating particles, in: Free Boundary Problems Theory and Applications, I. N. Figueiredo, J. F. Rodrigues, L. Santos (eds.), International Series of Numerical Mathematics 154, Birkhäuser, Basel, 2006, 107116.

[6] E. Caglioti, P.-L. Lions, C. Marchioro and M. Pulvirenti, A special class of stationary flows for two-dimensional Euler equations: a statistical mechanics description, Comm. Math. Phys. 143 (1992), 501-525.

[7] E. Caglioti, P.-L. Lions, C. Marchioro and M. Pulvirenti, A special class of stationary flows for two-dimensional Euler equations: a statistical mechanics description. II, Comm. Math. Phys. 174 (1995), 229-260. 
[8] P.-H. Chavanis, C. Rosier and C. Sire, Thermodynamics of self-gravitating systems, Phys. Rev. E 66 (2002), 036105.

[9] S. Luckhaus and Y. Sugiyama, Large time behavior of solutions in subcritical cases to degenerate Keller-Segel systems, Mathematical Modelling and Numerical Analysis 40 (2006), 597-621.

[10] A. Raczyński, Steady states for polytropic equation of self-gravitating gas, Math. Methods Appl. Sci. 28 (2005), 1881-1896.

[11] R. Schaaf, Stationary solutions of chemotaxis systems, Trans. Amer. Math. Soc. 292 (1985), 531-556.

[12] R. Schaaf, Global behaviour of solution branches for some Neumann problems depending on one or several parameters, J. Reine Angew. Math. 346 (1984), 1-31.

[13] M. Smoluchowski, Drei Vorträge über Diffusion, Brownsche Molekularbewegung und Koagulation von Kolloidteilchen, Phys. Zeit. 17 (1916), 557-571, 585-599.

[14] R. Stańczy, On some parabolic-elliptic system with self-similar pressure term, in: Selfsimilar solutions in nonlinear PDE's, P. Biler and G. Karch (eds.), Banach Center Publications 74, Inst. Math., Polish Acad. Sci., Warszawa, 2006, 205-215.

[15] R. Stańczy, Steady states for a system describing self-gravitating Fermi-Dirac particles, Differential Integral Equations 18 (2005), 567-582.

[16] R. Stańczy, Self-attracting Fermi-Dirac particles in canonical and microcanonical setting, Math. Methods Appl. Sciences 28 (2005), 975-990.

[17] R. Stańczy, On an evolution system describing self-gravitating particles in microcanonical setting, preprint, submitted.

[18] Y. Sugiyama, Global existence and finite time blow up for some degenerate Keller-Segel model, in: Variational Problems and Related Topics, Y. Naito and T. Suzuki (eds.), Sūrikaisekikenkyūsho Kōkyūroku 1464, Kyoto, 2005, 40-57.

[19] Y. Sugiyama and H. Kunii, Global existence and decay properties for a degenerate KellerSegel model with a power factor in drift term, J. Differential Equations 227 (2006), 333364 . 